\title{
Gaussian Kernel Density Estimator for Voxel Size Selection in Atom Probe Tomography
}

\author{
K. Kaluskar and K. Rajan \\ Department of Materials Science and Engineering/Iowa State University, Ames, Iowa, USA
}

Correct voxel size selection is crucial for many analyses done using Atom Probe Tomography (APT) data. Variation in density and concentration in a sample can be faithfully captured only if sampling of data is done so as to capture variation in underlying atomic distribution. To this end, in this work, we propose a voxel size selection method which is based on deciding optimal voxel size using Gaussian kernel density estimator and minimization of error [1].

Voxelization is used in APT to get local estimates of density and concentration. These estimates are then used to construct density/concentration profiles. Isoconcentration surfaces, for instance, are drawn based on these estimates and are used for visualization of chemical distribution, for identification of interesting features, and to measure gradient across interfaces [2, 3, and 4]. It is the usual practice to report voxel size and delocalization parameter while reporting these estimates. The challenges in voxel size selection has been long recognized in the APT community (see for example Hetherington and Miller [5], Hellman et.al.[6] and Torres et.al [7]). In this study we build on the prior work to develop mathematically objective selection criteria for voxel size selection using a non-parametric multivariate density function. Such functions are of value, especially when one needs to estimate the density directly from the data without assuming a particular form for the underlying distribution. This present study demonstrates the applicability of such methods in addressing in the voxel selection problem in atom probe tomography.

Voxel size should ideally be of same length scale as variation in density of underlying point cloud. However, it is often not possible to get a prior idea. Too large a voxel size will average out the variations and too small will be affected by statistical noise. In this method, we associate a cost function to some error measure for each voxel size. Voxel size for which cost function is minimized is chosen as final voxel size. We use Gaussian kernel density estimator due to its asymptotic nature. Delocalization is kept at 1.5 times the voxel size. Mean Square Error (MSE) is used as an error measure. It is dependent on variance and bias in the data and the estimate. The value of the method we present here is generic and can be applied with other density measures and other error measures.

Results will be presented for a simulated dataset where density variation is known and on an experimentally obtained dataset. Fig.1. shows the error measured for different voxel size for a subset of dataset. 


\section{References:}

[1] B. W. Silverman in "Density Estimation for Statistics and Data Analysis", (Chapman \& Hall/CRC Monographs on Statistics \& Applied Probability)

[2] A. Cerezo and M. G. Hetherington, J. Phys. Colloques 50 (1989), C8-523

[3] T. F. Kelly and M. K. Miller, Rev. of Sci. Instruments 78 (2007), 031101

[4] O. C. Hellman et al., Microsc. Microanal. 6 (2000), 437

[5] M. G. Hetherington and M. K. Miller., J. Phys. Colloques 50 (1989), C8-535

[6] O.C. Hellman, J. B. du Rivage, and D. N. Seidman, Ultramicroscopy 95 (2003), 199

[7] K. L. Torres et al., Ultramicroscopy 111 (2011), 464

[4] Support comes from Air Force Office of Scientific Research grants: FA9550-10-1-0256, FA955011-1-0158 and FA9550-12-0496; NSF grants: ARI Program CMMI-09-389018 and PHY CDI-09-41576; and Defense Advanced Research Projects Agency grant N66001-10-1-4004

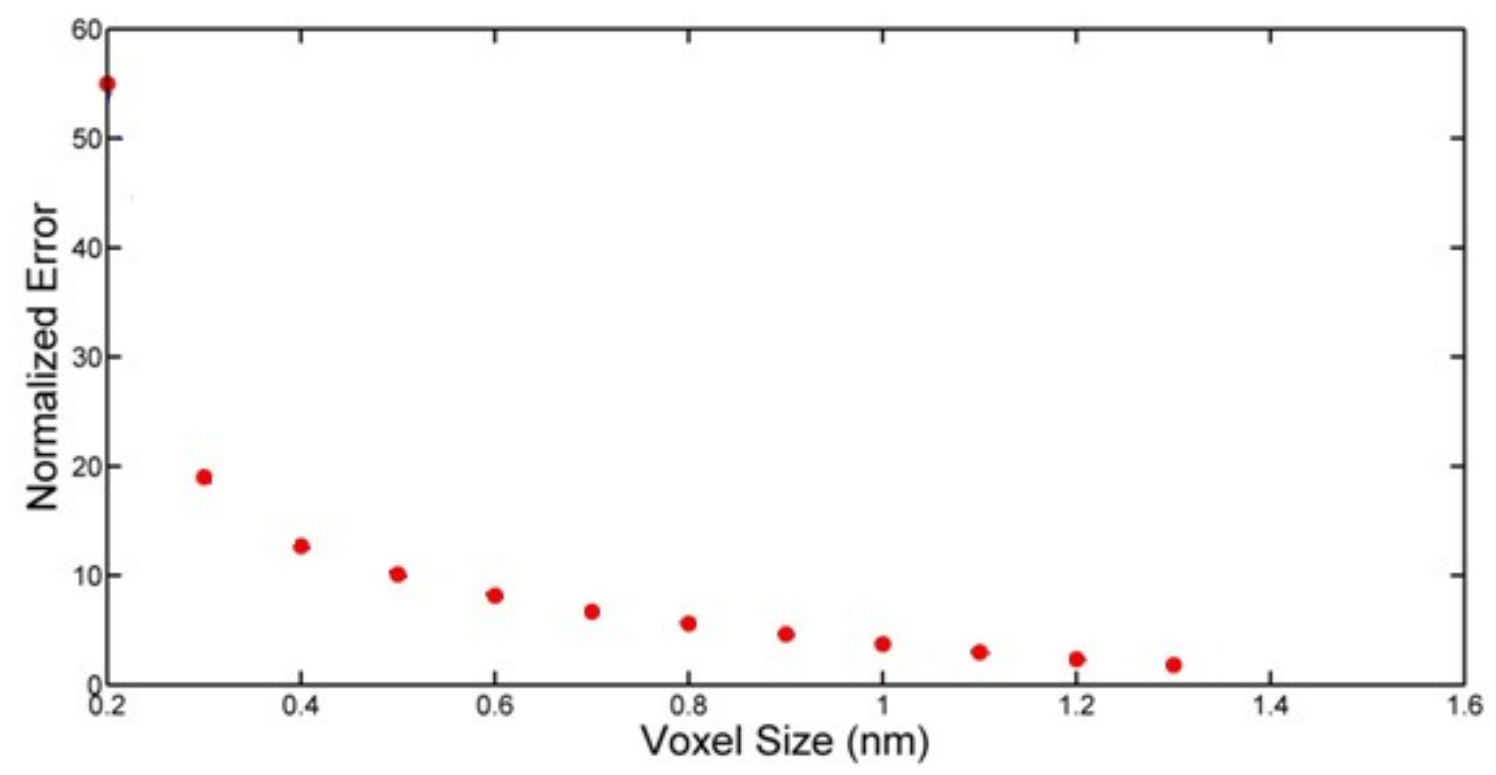

Figure 1 Mean Square Error at different Voxel sizes using Gaussian Kernel Density estimator. 